\title{
An improved particle swarm optimization based maximum power point tracking strategy with variable sampling time
}

\author{
Seyed Mohsen Mirhassani a,*, Sayedeh Zahra Mirbagheri Golroodbari ${ }^{a}$, \\ Sayedeh Mina Mirbagheri Golroodbari ${ }^{\mathrm{a}}$, Saad Mekhilef ${ }^{\mathrm{b}, 1}$ \\ a Department of Electrical of Engineering, University of Malaya, 50603 Kuala Lumpur, Malaysic \\ ${ }^{\mathrm{b}}$ Power Electronics and Renewable Energy Research Laboratory (PEARL), Department of Electrical of Engineering, University of Malaya, 50603 Kuala Lumpur, Malaysia
}

A R T I C L E I N F O

Article history:

Received 13 November 2013

Received in revised form 21 July 2014

Accepted 23 July 2014

Available online 28 August 2014

\section{Keywords:}

Maximum power point tracking

Particle swarm optimization

Partially shaded condition

Variable sampling time

\begin{abstract}
A B S T R A C T
This paper presents an improved maximum power point tracking (MPPT) strategy for photovoltaic (PV) systems based on particle swarm optimization (PSO). The capability of the PSO algorithm to cope with partially shaded conditions (PSCs) is the primary motivation of this research. Unlike conventional PSO-based MPPT systems, a variable sampling time strategy (VSTS) based on the investigation of the dynamic behavior of converter current is deployed to increase system tracking time. The performance of the proposed system is evaluated using MATLAB simulation and experimentation, in which a digital signal controller is used to implement the proposed algorithm on a real boost converter connected to a PV simulator. The main advantage of the proposed algorithm is fast and accurate performance under different conditions, including PSCs.
\end{abstract}

() 2014 Elsevier Ltd. All rights reserved.

\section{Introduction}

The increasing demand for energy and the limited capacity of fossil fuel resources have motivated numerous studies on alternative renewable energy resources in recent decades. Among the different renewable energy resources, photovoltaic (PV) solar energy, particularly in a grid-connected configuration, has attracted considerable interests because of its availability, low maintenance demand, and environment friendliness. Despite all its benefits, the high investment cost required by this technology makes extracting a large amount of available power from PV panels necessary. As a result, maximum power point tracking (MPPT) strategies should be deployed to ensure the tracking of the maximum power point (MPP) of nonlinear PV characteristics. The major challenges of MPPT lie in its dependence on the environmental parameters of the PV curve (i.e., temperature and insolation dependence) and in the local peaks created on the PV curve as a result of partially shaded PV panels (Fig. 2c) [1,2]. Partially shaded condition (PSC) will be discussed in the next section.

Several MPPT strategies have been suggested in literature. These strategies can be classified into off-line and on-line approaches.

\footnotetext{
* Corresponding author. Tel.: +60 173784010 .

E-mail addresses: mohsen.mirhassani@gmail.com (S.M. Mirhassani), saad@um. edu.my (S. Mekhilef).

${ }^{1}$ Tel.: +60 379676851 ; fax: +60379675316

Off-line approaches estimate the location of the MPP based on the open circuit voltage or short circuit current of the panel and on the standard test information provided by the manufacturer $[3,4]$. However, the nonlinear dependence of PV characteristics on cell temperature and solar insolation is a major road block against the functionality of these approaches. Such dependence results in significant errors in MPPT in some situations. On-line approaches focus on the information being acquired while energy is transferred from the PV, such that any estimation based on standard test information will be unnecessary, and the controller easily adapts itself to any variation of the aforementioned parameters. Among the on-line approaches, perturb and observe (P\&O) [5-7] and incremental conductance (IC) [8-10] are two well-known MPPT strategies. In addition to imposing an inevitable tradeoff between tracking speed and fluctuation of extracted power, these strategies cannot differentiate between global and local MPP when PSC causes a multi-peak $P-V$, thus resulting in the tracking of a local peak that may not be the global MPP. Furthermore, typical P\&O strategies suffer from the loss of correct direction of the MPP location in case of rapid changes in solar insolation [11]. From the control perspective, MPPT strategies can also be classified into direct [e.g., hill climbing ( $\mathrm{HC})$ ] and indirect approaches (e.g., P\&O and IC). Direct approaches take control of the duty cycle of the converter switch, whereas the output of the indirect algorithm is a reference value for the PV voltage or current. In this case, an additional control loop [i.e., proportional integral derivative (PID) controller] is needed to adapt the duty cycle to 


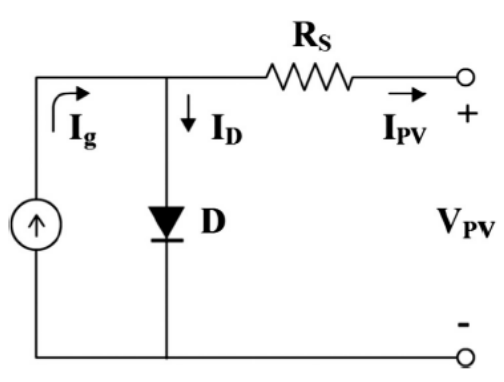

Fig. 1. Equivalent circuit of the PV cell.

the reference value, resulting in additional problems associated with controllers such as tuning and steady state error. To address such problems, several works are carried out to employ fuzzy logic controller [12-14]. However, the computational burden caused by four stages of fuzzification, rule base, inference mechanism and defuzzification leads to sluggish performance and lower tracking speed of the system.

Apart from typical MPPT strategies, novel approaches can be found in the literature [15]. Proposes a system which performs a modified P\&O based MPPT only by observing the modules voltage. As a result current sensor which is a key part to observe power in typical MPPT approaches is removed from the system causing significant simplification. However, the need for sequential MPP leads to a sluggish performance and the problem with the PSC still exists. For the communication purpose between modules and processor, Zigbee wireless network is used which is another nove aspect of this paper. A genetic algorithm (GA) based MPPT strategy is presented in [16] where GA is employed to estimate the MPP's current $\left(I_{M P P}\right)$ based on solar radiation and module surface temperature. In addition to neglecting PSC the need for radiation and temperature measurement makes the overall system more complicated. Application of neural network (NN) in MPPT is investigated in [17] where coefficients of a proposed cubic equation are adapted by an NN to calculate MPP for different solar radiation. The result is a fast system able to track MPP under fluctuating insolation however the effect of PSC is neglected.

To cope with MPPT under PSC, [18] suggests a typical P\&O approach associated with a line search algorithm, called DIRECT, which is applied to the $I-V$ curve to identify the PSC in the first step and to track the global peak (GP) in the second step. Aside from the complexity of this two-step algorithm, the need to identify the PSC poses a risk of failure. Another line search algorithm is suggested in [19]. This algorithm employs the Fibonacci sequence instead of the DIRECT method. In addition to the aforementioned problems associated with the P\&O approach, this algorithm cannot guarantee that the GP can be found under all conditions. To address this problem [14], presents a modified Fibonacci search algorithm which performs a wide range of search irrespective of original operating point. However wider range of search leads a bigger tracking time. In [20], the authors proposed a two-stage algorithm to search for the possible GP location and accurately track the global MPP using the P\&O approach. The search algorithm is based on the fact that $V_{M P P}$ of each string is usually located somewhere near $80 \%$ of the open circuit voltage of this string. The algorithm appears to work properly. However, the necessity of using an indirect approach to examine all possible spots of the GP causes the tracking time to reach as long as $4 \mathrm{~s}$.

The idea of using particle swarm optimization (PSO) for MPPT application was recently proposed [11,21-24]. PSO-based algorithms show several advantages over other algorithms used to cope with the PSC, including the lack of a need to identify the PSC, capability for use in single-stage configuration, and fast and simple structure. However, owing to the need to perturb large and sudden changes in the duty cycle, a large $\mathrm{ST}^{2}$ must be considered to ensure that the measurement parameters [i.e., power and voltage of the panel] are not in the transient state. Consequently, to avoid sluggish performance, only a small number of iterations are allowed, which results in inaccurate GP tracking. Ishaque and Salam [21] propose eliminating the randomness of the algorithm to achieve faster searches on the PV curve. An additional HC method is also considered to accurately track the GP, resulting in a higher degree of complexity. A modified PSO-based MPPT is proposed in [23]. Unlike conventional PSO algorithms, the three basic tuning parameters [i.e., inertia factor and acceleration coefficients] are considered variable to accelerate convergence. The suggested variable PSO strategy accomplishes particle convergence fairly well, but requires at least 26 iterations with a ST of $0.2 \mathrm{~s}$, thus resulting in sluggish performance.

Variable ST is used in networked control system to address instability caused by time delays and possibility of data loss in wireless feedback control loops [25-28] while in MPPT algorithms available in the literature, ST is normally considered constant. In this paper, a variable sampling time PSO algorithm is proposed to accelerate the tracking procedure and allow for a greater number of iterations. The performance of the proposed algorithm is evaluated using simulation and experiments. The remainder of this paper is organized as follows: Section 'Characteristics of the PV under PSC' explains the basic modeling of a PV cell and discusses the effect of PSC on the PV curve. Section 'Proposed PSO method' presents the basic knowledge on PSO and then discusses the application of PSO in MPPT. Three important factors in the design of the proposed PSO algorithm, namely, number of particles, convergence, and sampling time, are investigated in this section as well. The flowchart of the system, initialization, and reinitialization are also explained in this section. The section ends by highlighting the advantages of the proposed method. Sections 'Simulation results' and 'Experimental results' validate the proposed algorithm under uniform insolation, fast transient changes in insolation, and PSC. The performance of the proposed system is also validated under PSC by the experimental results in Section 'Experimental results'. Finally, the conclusion is provided in Section 'Conclusion'.

\section{Characteristics of the PV under PSC}

\section{Modeling of a PV cell}

A PV cell can be modeled by current source $I_{g}$, diode $D$, and series resistance $R_{5}$. The model is illustrated in Fig. 1. The output current of the PV cell is the difference between the photocurrent $I_{g}$ and the diode current $I_{D}$. This current can thus be expressed as follows:

$I_{P V}=I_{g}-I_{s}\left(\exp \left(\frac{q\left(V_{P V}+I_{P V} \cdot R_{s}\right)}{n k T}\right)-1\right)$

where $n$ is the diode ideality factor, $k$ is Boltzmann's constant, $T$ is the temperature in Kelvin, $q$ is the electron charge, $R_{s}$ is the equivalent series resistance, and $I_{s}$ is the saturation current.

\section{Effect of PSC}

The output voltage of a PV cell is quite low (almost $0.6 \mathrm{~V}$ ), to increase the output voltage a string of series connected PV cells is needed. Meanwhile, if a part of the string is shaded, the voltage generated by the shaded cells will be lower than that generated by

${ }^{2}$ Sampling time. 

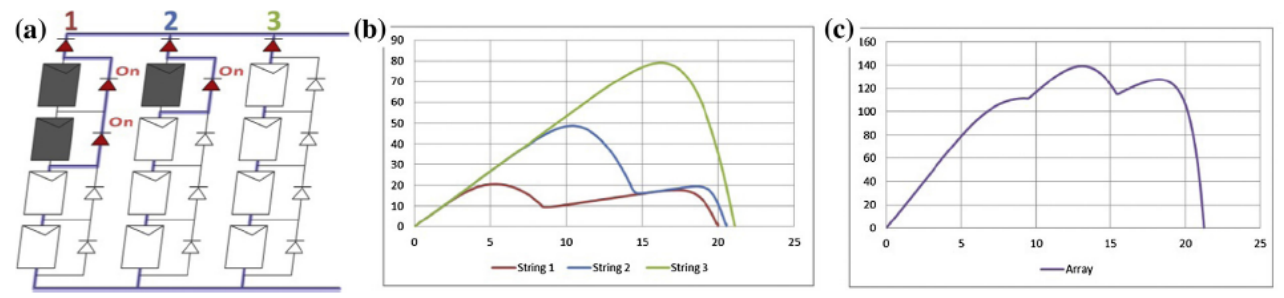

Fig. 2. (a) PSC in a $4 \times 1$ PV array: insolation considered for un-shaded modules, $1000 \mathrm{~W} / \mathrm{m}^{2}$ and insolation considered for shaded modules, $350 \mathrm{~W} / \mathrm{m}^{2}$, (b) $P-V$ curves of each string; and (c) $P-V$ curve of the array.

un-shaded cells, which results in the consumption of a part of the generated power by shaded cells. This situation not only results in significant power loss in the PV array, but also causes hot spots in the location of shaded cells, consequently reducing the lifetime of PV cells. To avoid this problem, bypass diodes are deployed to bypass the shaded cells. The operation of bypass diodes in a PV array with parallel strings is shown in Fig. 2a. Although the diodes save the shaded cells from the aforementioned problem, they change the PV characteristics and cause a two-peak curve. Difficulty arises when several strings are connected in parallel to supply a higher current, as demonstrated in Fig. 2b. Depending on the number of shaded cells, different PV curves are generated by each string. These multi-peak PV curves are then combined because of the parallel connection, which gives rise to the multipeak curve shown in Fig. 2c.

\section{Proposed PSO method}

PSO method

PSO is a method classified as an evolutionary algorithm modeled after the behavior of birds. The PSO comprises a swarm of particles, and each particle suggests a candidate solution for the optimization problem. The basic idea behind PSO is to emulate the success of all particles in the population. In other words, the position of the particle is affected by the agent position, which suggests the best solution through the current particles $P_{\text {best }}$, as well as the best solution suggested through the entire population $G$. The particle position $x_{i}$ is adjusted using:

$x_{i}^{k+1}=x_{i}^{k}+v_{i}$

where the velocity component $v_{i}$ represents the step size and is calculated by using the following expression:

$v_{i}^{k+1}=\omega v_{i}^{k}+\boldsymbol{c}_{1} \boldsymbol{r}_{1}\left(P_{\text {besti }}-x_{i}^{k}\right)+\boldsymbol{c}_{2} \boldsymbol{r}_{2}\left(G-x_{i}^{k}\right)$

where $\omega$ is the inertial weight, $\boldsymbol{c}_{\mathbf{1}}$ and $\boldsymbol{c}_{\mathbf{2}}$ are the acceleration coefficients, $\boldsymbol{r}_{\mathbf{1}}$ and $\boldsymbol{r}_{2}$ are random values that belong to the interval of $[0,1], P_{\text {best }}$ is the best position of particle $i$, and $G$ is the best position in the entire population.

\section{Application of PSO in MPPT}

To deploy the PSO for MPPT application, a typical MPPT method should be employed. Most studies employed the HC method for two reasons: this method has a simple structure and directly controls the duty cycle, thus eliminating the need for an additional PID controller [11]. The algorithm of the HC method is shown in Fig. 3. First, an initial value for the duty cycle $(D)$ is selected. The value of $D$ is then changed with respect to the variation of power. In other words, as long as the power is increasing, the sign of the "slope" parameter remains unchanged [i.e., positive]. Once the power starts to decrease, the sign is changed to the opposite [i.e., negative].

To implement the PSO, $D$ is defined as a particle. Therefore, (2) can be rewritten as follows:

$D_{i}^{k+1}=D_{i}^{k}+v_{i}$

The aim of the MPPT program is to find the MPP. Thus, the objective function of the PSO algorithm would be:

$P\left(d_{i}^{K}\right)>P\left(d_{i}^{K-1}\right)$

where $P$ represents power, $d$ is the duty cycle, $i$ is the number of current particles, and $K$ shows the number of iterations.

Design of the proposed algorithm

The number of particles is a very important factor in PSO design. After applying each duty cycle, we must wait for the transient condition to settle; the resulting large number of particles will increase the tracking time of the MPP. In this paper, three particles are considered. The functionality of the PSO algorithm with three particles is illustrated in Fig. 4. Taking into considering (3), the velocity expression comprises three terms. The first term is called inertia force, which is affected by the former velocity factor. The second and third terms are affected by the distance of the particles from the best particle among the three $\left(D_{\text {besti }}\right)$ and the best particle so far $(G)$ respectively. A greater distance indicates greater potential. The two potential factors are then multiplied by the random values to allow for a better search of PV characteristics. As demonstrated in Fig. 4, inertia force may be applied in the direction of G (agent 1) or the opposite direction (agent 3) to allow for better convergence. Furthermore, $D_{\text {best }}$ is affected only by two forces. Thus, a smaller velocity for this particle prevents it from losing the best particle position $(G)$ while allowing for more explorations because of the corresponding random factor. In sum, three coefficients have to be tuned: $\omega, c_{1}$, and $c_{2}$. Selecting a large value for the inertia factor results in long tracking time, whereas a small value may cause convergence problems, which will be explained later in this section. Large values for the other two coefficients result in long tracking time and convergence problems, whereas small values can cause algorithm failure to find the MPP. Owing to the randomness of the algorithm, finding a classified procedure for tuning the coefficients is difficult. Thus, the coefficients are usually tuned through experiments.

Another important factor is particle convergence because the duty cycle cannot accept any value outside the interval $[0,1)$. Thus, the particles have to be converged in this interval; otherwise, the system will not find the MPP. To ensure that the particle values do not exceed the interval, the speed parameter $\left(v_{i}\right)$ has to be controlled. In [21], the authors suggest limiting the speed to a maximum value $(0.035)$. However, this method cannot differentiate between particles, which results in a speed greater than the 


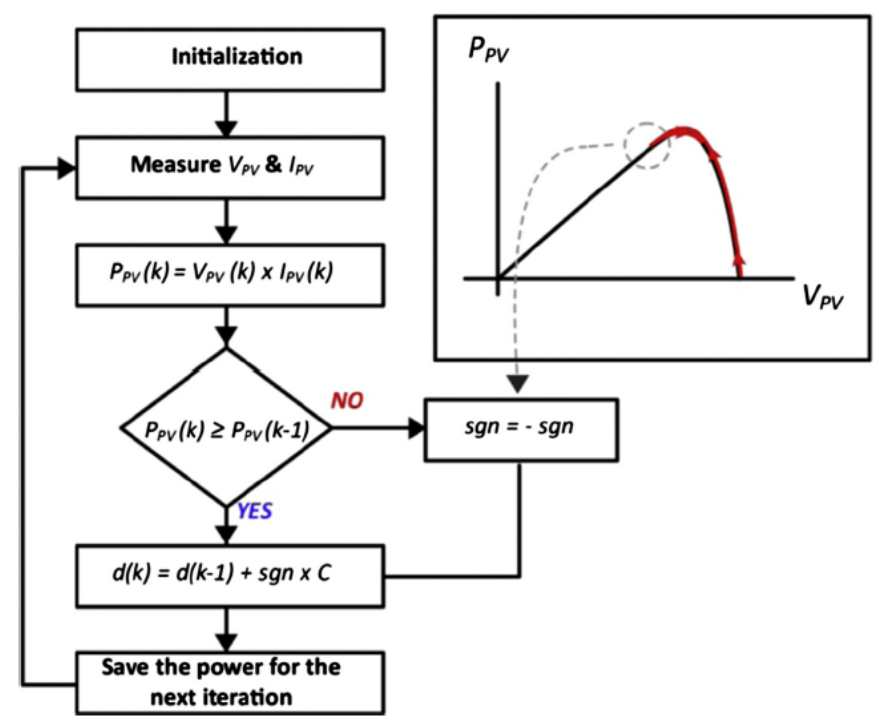

Fig. 3. HC algorithm.

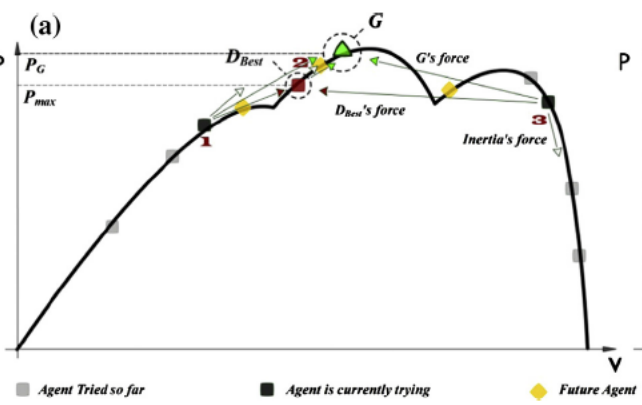

(b)

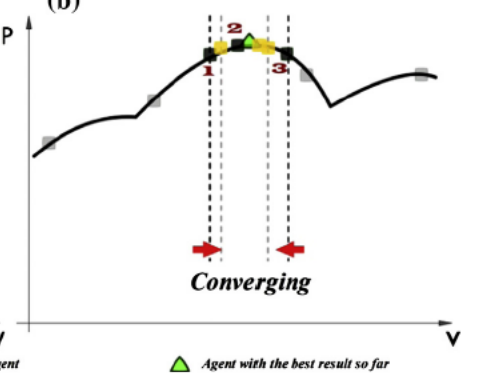

Fig. 4. PSO algorithm with three particles (a) at the middle of the execution time and (b) at the end of the execution time.

maximum value and causes longer tracking time. In this study, the inverse tangent function is used to retain the speed within a safe margin. While allowing for the implementation of greater speed terms with respect to the distance of the particle to the desired value, this function ensures that the value generated by the random coefficients is within the interval. The speed expression is described below. To reduce computation time, the function can be estimated by a lookup table.

$v_{i}^{k+1}=\frac{2}{\pi} \arctan \left(\omega v_{i}^{k}+\boldsymbol{c}_{1} \boldsymbol{r}_{1}\left(D_{\text {besti }}-D_{i}^{k}\right)+\boldsymbol{c}_{2} \boldsymbol{r}_{2}\left(G-D_{i}^{k}\right)\right)$

where $D_{\text {besti }}$ is the duty cycle that results in the maximum power among the three particles, and $G$ is the duty cycle that results in the maximum power through the entire population.

The last factor to be considered is sampling time. After applying each particle, the controller should wait for the power to settle. Thus, after applying each duty cycle, a delay occurs during the execution of the MPP program. Most authors considered a FSTS

\footnotetext{
${ }^{3}$ Fixed Sampling Time Strategy.
}

[11,21-24]. However, the settling time of the system varies with the step size. For instance, the settling time of the power after perturbing $20 \%$ change in the duty cycle is not the same as the value for perturbing only $5 \%$ change. By contrast, as the program loop executes, the particles become close to one another (Fig. 4b), which indicates that applying a variable sampling time strategy (VSTS) can accelerate the tracking procedure significantly.

\section{State space model of the system}

To study the effect of step size on settling time, the state space (SS) model of the system will be derived in this section. The schematic of the system under study is demonstrated in Fig. 5. To derive a linearized SS model, the PV is considered as a constant independent current source. The SS model can be derived easily by applying KVL in loops 1 and 2 and KCL in nodes 1 and 2 in the two following instances:

- The switch is on and the diode is off $\left(T_{o n}\right)$

- The switch is off and the diode is on $\left(T_{\text {off }}\right)$ 
$L 1$

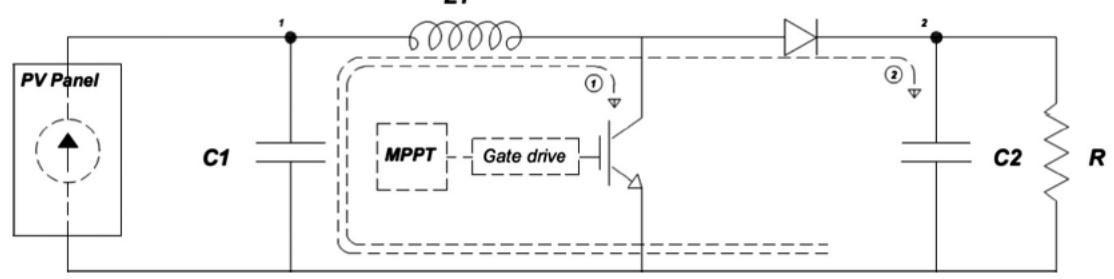

Fig. 5. Schematic of the system under study.

By considering the capacitor voltages $V_{c 1}$ and $V_{c 2}$ and the inductor current $i_{L}$ as the SS variables, the model can be described as follows:

$$
\left[\begin{array}{c}
\frac{d i_{1}}{d t} \\
\frac{d v_{C 1}}{d t} \\
\frac{d v_{C 2}}{d t}
\end{array}\right]=\left[\begin{array}{ccc}
0 & \frac{-1}{C_{1}} & 0 \\
\frac{1}{L} & 0 & \frac{-(1-D)}{L} \\
0 & \frac{1-D}{C_{2}} & \frac{-1}{R C_{2}}
\end{array}\right]\left[\begin{array}{c}
i_{L} \\
v_{C 1} \\
v_{C 2}
\end{array}\right]+\left[\begin{array}{c}
\frac{1}{C_{1}} \\
0 \\
0
\end{array}\right] I_{\text {in }}
$$

where $C_{1}$ and $C_{2}$ are the input and output capacitors respectively (see Fig. 5), $L$ is the inductor, $R$ is the load resistance, and $D$ represents the duty cycle of the converter. The input current is assumed to be a constant value, such that the settling time of the power is the same as that of the input voltage. To investigate input voltage behavior, the following values for matrix $C$ of the SS model are defined:

$$
C=\left[\begin{array}{lll}
0 & 1 & 0
\end{array}\right]
$$

By considering (7) and (8), the value of the SS model parameters $\mathrm{A}, \mathrm{B}$, and $\mathrm{C}$ are known. Considering constant input, zero value can be assumed for the $D$ matrix in this level. The response of the SS model to the different steps of the duty cycle is demonstrated in Fig. 6. Significant changes in the duty cycle require a longer time to settle. As a result, shorter delay in perturbation of new duty cycle can be considered when there is only a small variation in the value of duty cycle. Considering the fact that as the PSO algorithm is executed further position of particles come close to one another, the VSTS can provide a significant acceleration in tracking procedure. Notably, for the proposed algorithm and for the inertia and acceleration coefficients used in the system, the possibility of perturbing steps greater than $30 \%$ is negligible. The ST, therefore, is defined with the following rule:

$$
\{\Delta D \leqslant 0.08 \quad S T=0.003 \mathrm{~s}
$$$$
\{D>0.08 \text { ST }=0.006 \mathrm{~s}
$$

The flowchart of the proposed system is illustrated in Fig. 7. The program starts with the initialization step, which defines the appropriate values for the particles and speed terms to ensure that the program adequately explores the PV characteristics. After the first step, the program starts an infinity loop to find the MPP. This loop will be broken after finding the MPP to avoid unnecessary fluctuations in power.

Structure and properties of the proposed system

To ensure that system performance is fast and accurate, the starting point of the program has to be considered. Execution of the MPPT program starts by satisfying either of the following two conditions:

- When the system has just turned on and no valid record of the last MPP tracked (initialization) exists

- When the MPP tracked by the system is no longer valid because of a significant change in the insolation (reinitializing)

(1) Initialization: This step is usually performed by using random base functions [23]. However, initialization serves an important function in system tracking time. Convergence time of

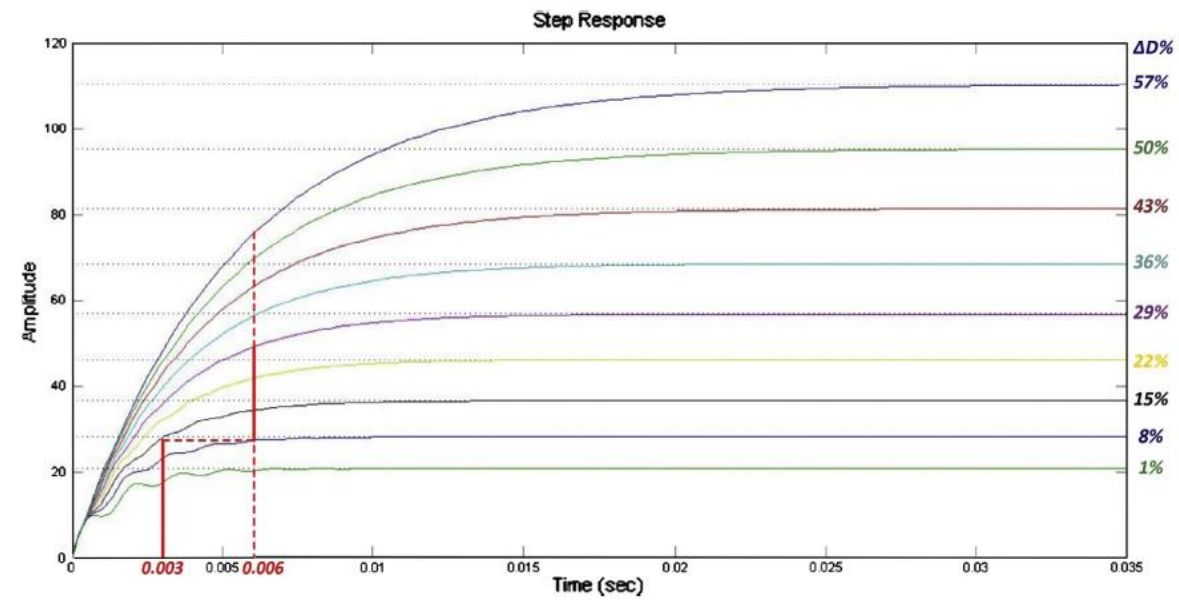

Fig. 6. Step response to the different changes in the duty cycle. 


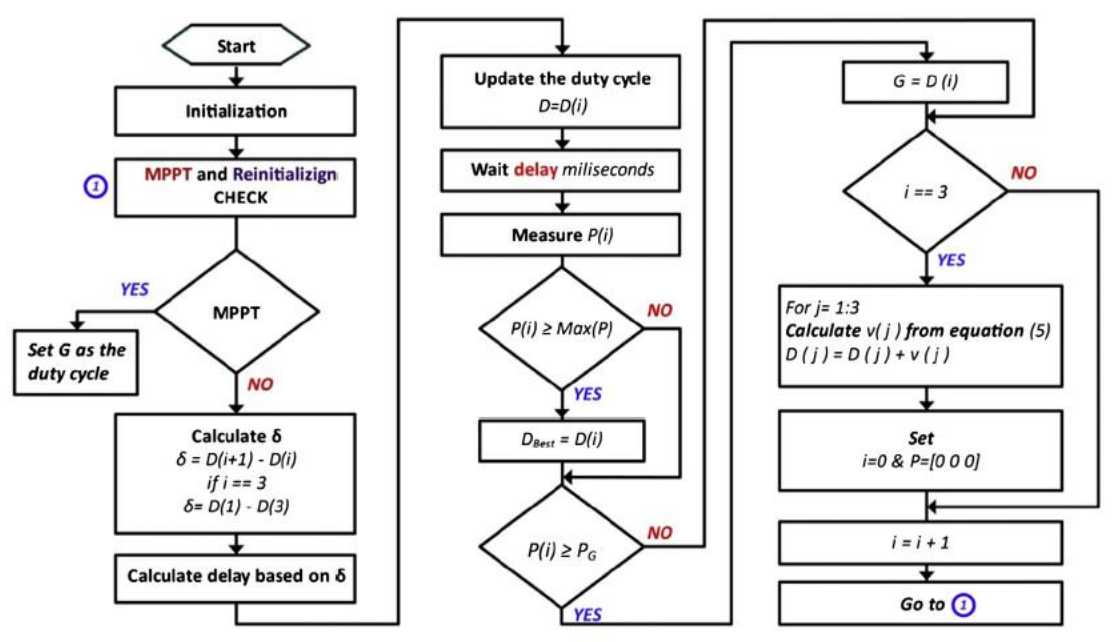

Fig. 7. Flowchart of the proposed method.

the particles and adequate exploration on the PV curve are two important factors to be considered. In this study, small and close particles with positive speed terms are considered as a result of most possible insolation conditions, such that the initial operating point is located at the right side of the MPP. Although the initial locations of the agents are not vital for the correct operation of proposed algorithm, this factor has considerable influence on tracking time and power fluctuations during the tracking procedure.

(2) Reinitialization: When the system tracks the MPP, particles have values close to one another and speed terms that are almost zero. Consequently, if a change in the PV characteristic occurs, the system may be incapable of tracking the new MPP because of the zero value of speed terms. Meanwhile, some information derived by the program (i.e., global MPP: $P_{G}$ and corresponding duty cycle $G$ ) is no longer valid, which may result in the tracking of a wrong point on the PV curve. To avoid such problems, changes in PV characteristics should be determined and the system should be reinitialized. A change in PV curve may occur either during or after MPPT. In both cases, a simple verification of the particles near $G$ can determine changes in insolation, such that the system can reinitialize if the following conditions are satisfied:

$|d(i)-G|<\Delta D_{\min }$
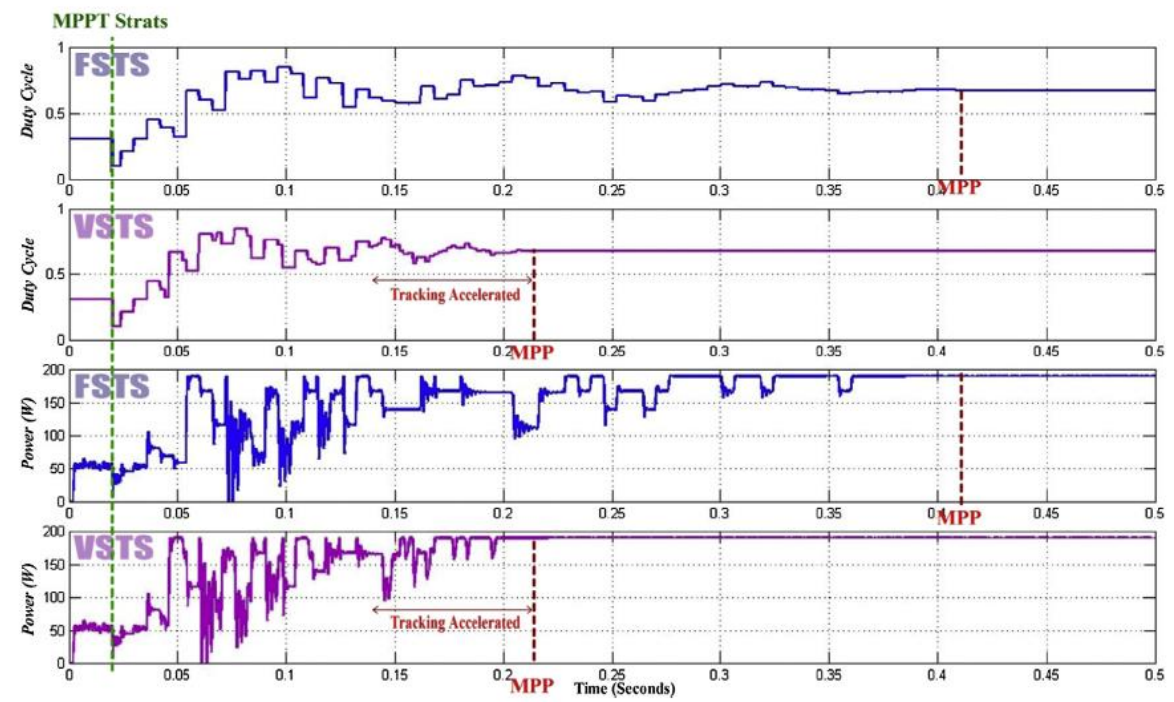

Fig. 8. Comparison between the VSTS and FSTS.

Link to Full-Text Articles :

http://www.sciencedirect.com/science/article/pii/S0142061514005183 